\title{
Barcoding the Dendrobium (Orchidaceae) Species and Analysis of the Intragenomic Variation Based on the Internal Transcribed Spacer 2
}

\author{
Xiaoyue Wang, Xiaochen Chen, Pei Yang, Lili Wang, and Jianping Han \\ Institute of Medicinal Plant Development, Chinese Academy of Medical Sciences, Peking Union Medical College, Beijing, China \\ Correspondence should be addressed to Jianping Han; jphan@implad.ac.cn
}

Received 8 June 2017; Revised 17 August 2017; Accepted 13 September 2017; Published 17 October 2017

Academic Editor: Chiu-Chung Young

Copyright (C) 2017 Xiaoyue Wang et al. This is an open access article distributed under the Creative Commons Attribution License, which permits unrestricted use, distribution, and reproduction in any medium, provided the original work is properly cited.

\begin{abstract}
Many species belonging to the genus Dendrobium are of great commercial value. However, their difficult growth conditions and high demand have caused many of these species to become endangered. Indeed, counterfeit Dendrobium products are common, especially in medicinal markets. This study aims to assess the suitability of the internal transcribed spacer 2 (ITS2) region as a marker for identifying Dendrobium and to evaluate its intragenomic variation in Dendrobium species. In total, 29,624 ITS2 copies from 18 species were obtained using 454 pyrosequencing to evaluate intragenomic variation. In addition, 513 ITS2 sequences from 26 Dendrobium species were used to assess its identification suitability. The highest intragenomic genetic distance was observed in Dendrobium chrysotoxum (0.081). The average intraspecific genetic distances of each species ranged from 0 to 0.032 . Phylogenetic trees based on ITS2 sequences showed that most Dendrobium species are monophyletic. The intragenomic and intraspecies divergence analysis showed that greater intragenomic divergence is mostly correlated with larger intraspecific variation. As a major ITS2 variant becomes more common in genome, there are fewer intraspecific variable sites in ITS2 sequences at the species level. The results demonstrated that the intragenomic multiple copies of ITS2 did not affect species identification.
\end{abstract}

\section{Introduction}

Dendrobium is one of the three largest genera of the Orchidaceae family and comprises more than 1,000 species distributed throughout the Asian tropical and subtropical regions as well as Oceania, with 78 species of this genus recorded in China alone [1]. The flowers of Dendrobium come in a rich variety of colors and shapes, and in recent years they have increased significantly in commercial value as ornamental flowers. In addition, Dendrobium is also well known for its medical value. In fact, one of the earliest records of Orchidaceae plants in ancient Chinese literature is Shen Nong's classic herbal text written approximately 1,500 years ago. Approximately 33 species of Dendrobium are used as clinical medications [2], including Dendrobium officinale, also known as "Tie Pi Feng Dou," and Dendrobium nobile, also known as "Jin chai shi hu," as described in the Chinese Pharmacopoeia. Each year, large numbers of Dendrobium species are needed for both the flower and medicinal markets.
Adulterants and substitutes have become popular in the markets, especially for medicinal purposes. Thus, an effective method of species identification is very necessary.

In eukaryotic genomes, rDNA arrays are often present in hundreds of copies, with copy number varying among different species [3-5]. As a tool to study evolution, the rDNA copy number per genome and sequence variation between species can be used to study phylogenetic relationships and biodiversity [4]. The internal transcribed spacer (ITS) is part of a multicopy gene that encodes ribosomal RNA subunits in all eukaryotic genomes. ITS regions have been used to study biodiversity in bacteria [6], insects [7], marine organisms [8-10], and plants [11], as well as many others. Due to their powerful discriminatory ability and stability among Dendrobium species, rDNA sequences have been used for identification and classification purposes [12,13]. Among the numerous Dendrobium species, D. officinale has received the greatest amount of attention due to its high medicinal value in China. Ding et al. established a database that included 21 Dendrobium species labelled "Feng Dou" herbs on the market and 
proposed that rDNA ITS sequences could be used to identify Dendrobium species with high accuracy [14]. Indeed, Zhang et al. accurately identified $D$. officinale from its adulterants using full-length ITS regions [15]. Furthermore, Li et al. performed phylogenetic analyses and identified Dendrobium species using rDNA ITS sequences, and their classification based on ITS sequences was identical to traditional classifications for most species [16].

ITS2 is commonly used to infer phylogenetic relationships and has been employed as a DNA barcode for identification purposes. The genes in this region are thought to have evolved in concert, leading to a homogenization of all copies of this gene across the genome $[17,18]$. To date, the ITS2 region has been used to identify plants [19-21], fungi [2224], and insects [25]. Although ITS/ITS2 is extremely useful for both species identification and phylogenetic analyses, it does have drawbacks. One significant problem is the fact that it is present in multiple copies in the genome. Phylogenetic studies typically use consensus sequences that average over all copies in a genome, thereby concealing most intragenomic variation. Indeed, the intragenomic variation and intraspecies divergence in ITS2 present significant challenges for genetic diversity analyses and species identification. In contrast, the evaluation of ITS2 sequences for identification and phylogenetic purposes might prove useful for deep research into intragenomic and intraspecific diversity. While intraspecific divergence in Dendrobium has been studied, the issue of intragenomic diversity revealed by multicopy has received increased attention due to the development of next-generation sequencing technology. Here we used pyrosequencing to sequence 18 selected species of Dendrobium to perform ITS2 intragenomic diversity analysis. Intraand interspecific variations among different species were also evaluated using ITS2 sequences in 26 species of Dendrobium. Our results indicate that the ITS2 region is a valuable tool for identifying species and analyzing phylogenetic relationships.

\section{Material and Methods}

2.1. Sampling, DNA Extraction, PCR Amplification, and Sequencing. Fresh leaves and stems of plants of the genus Dendrobium were obtained from different locations (see Appendix S1, in Supplementary Material available online at https://doi.org/10.1155/2017/2734960). Samples were dried at a temperature of $45^{\circ} \mathrm{C}$ prior to genomic DNA extraction. DNA extraction, PCR amplification, and sequencing were performed as described in previous studies [26, 27]. Approximately $15 \mathrm{mg}$ of dried leaves or $20 \mathrm{mg}$ of dried stems was ground for two minutes (30 revolutions/second) in a FastPrep bead mill (MM400, Retsch, Haan, Germany). DNA was extracted using the Plant Genomic DNA Kit (Tiangen Biotech Co., Beijing, China). Universal primers for the ITS2 region (ITS2F/3R) were used for amplification [27]. Sequencing of the PCR products was performed bidirectionally with the same primers used for the PCR amplification using a 3730XL sequencer (Applied Biosystems, Foster, California, USA). The intragenomic data used were from a previous study by our group [28]. Other sequences were obtained from GenBank (see Appendix S2). Twenty-six Dendrobium species with more than ten sequences each were selected for identification analysis.

2.2. Data Analysis. ITS2 sequences in this study were subjected to hidden Markov model (HMM) [29] analysis to remove the conserved $5.8 \mathrm{~S}$ and $26 \mathrm{~S}$ rRNA genes. Intragenomic and intraspecific Kimura 2-parameter distances were computed using the MEGA 5.2.2 software [30] (default parameters: variance estimation method: bootstrap method; number of bootstrap replications: 1000; model: Kimura 2parameter model; substitutions to include transition + transversions; rates among sites: uniform rates; and gaps/missing data treatment: complete deletion). Mega 5.2.2 was used to construct a neighbor-joining tree (default parameters: test of phylogeny: bootstrap method; number of bootstrap replications: 1000; model: Kimura 2-parameter model; substitutions to include: transition + transversions; rates among sites: uniform rates; and gaps/missing data treatment: complete deletion).

\section{Results}

3.1. Intragenomic Variations in 18 Species of Dendrobium. We first investigated the levels of intragenomic variation in the ITS2 regions of 18 Dendrobium medicinal materials. A summary of the intragenomic variation is provided in Table 1. In total, 29624 ITS2 copies from 18 species were obtained using 454 pyrosequencing in a previous study by our group. The numbers of ITS2 variant copies are 4 to 55 , with $D$. crepidatum having the least and D. chrysotoxum having the most. Here, we refer to variants with an emergence frequency above $1 \%$ as "major variant(s)." Major variants representing more than $90 \%$ of ITS2 sequences were found in D. crepidatum (p1-1), D. aphyllum (P2-1), D. devonianum (p31), D. officinale (p4-1), D. trigonopus (p16-1), D. gratiosissimum (p15-1), D. capillipes (p13-1), and D. denneanum (p5-1), which were present at $99.27 \%, 95.87 \%, 95.43 \%, 93.89 \%, 93.34 \%$, $93.23 \%, 93.03 \%$, and $91.50 \%$ of total sequences, respectively. The major variants of each species were used to calculate the intragenomic genetic distance (IG-GD). The highest IGGD was found in D. chrysotoxum (0.081). In contrast, the genetic distance in five species (D. officinale, D. crepidatum, D. aphyllum, D. wardianum, and D. trigonopus) was zero, indicating minimal intragenomic diversity. D. williamsonii showed the most intragenomic variant patterns of the 18 study species, showing 52 distinct major variant patterns that ranged in prevalence from $25.82 \%$ to $1.1 \%$. The GC content of the variants from these 18 species ranged from $47.35 \%$ to $56.00 \%$. No obvious differences were observed in the length of major variants.

In this study, the $D$. officinale sample received a total of 2554 reads of 454 pyrosequencing representing ten different variant patterns. The most common major variant represented $93.89 \%$ of the ITS2 sequences in the entire genome. After alignment, the consensus sequence of the ten variants from the $D$. officinale genome was 246 bp in length, with 13 variable sites, including two INDELS. The dominant 


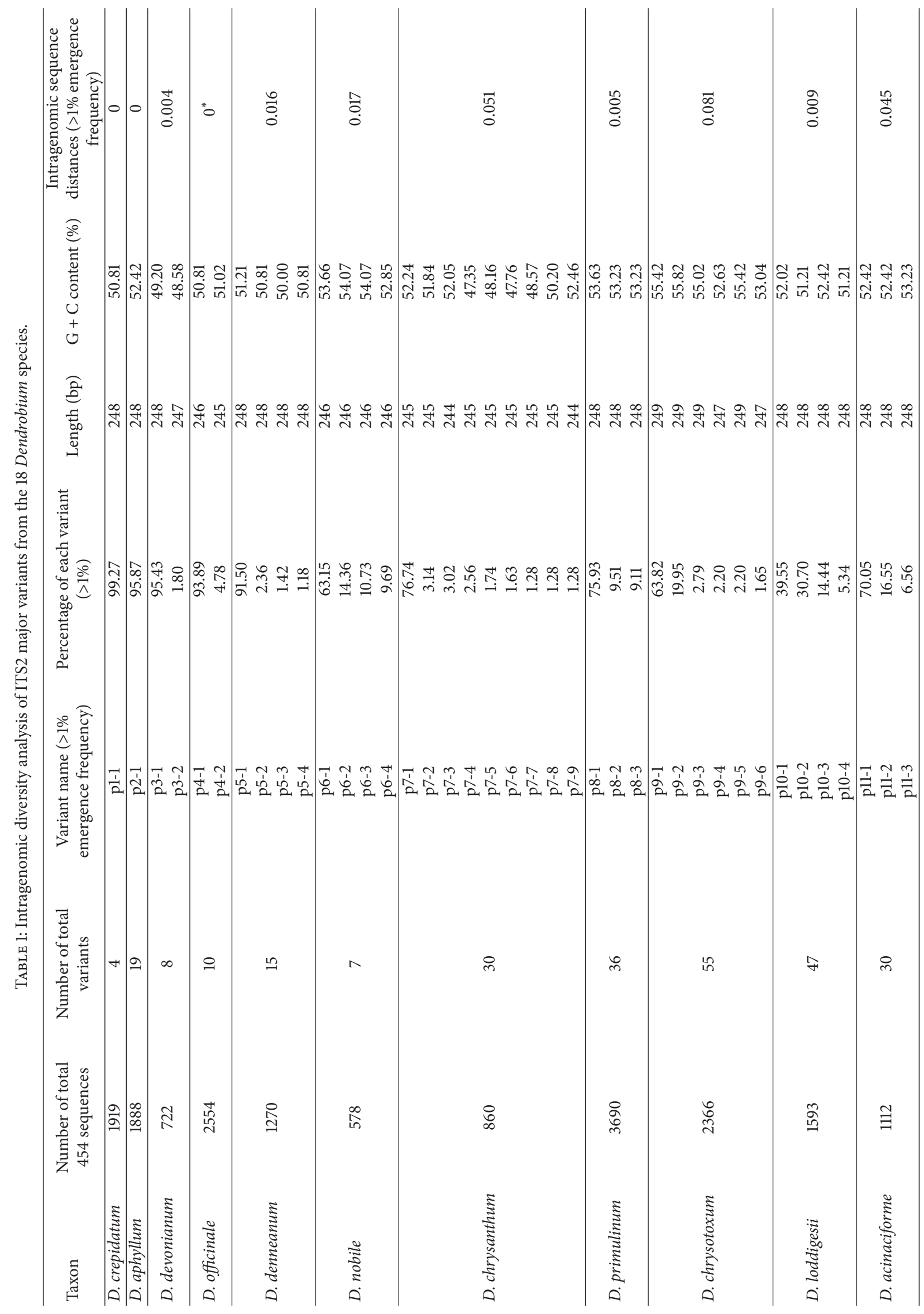




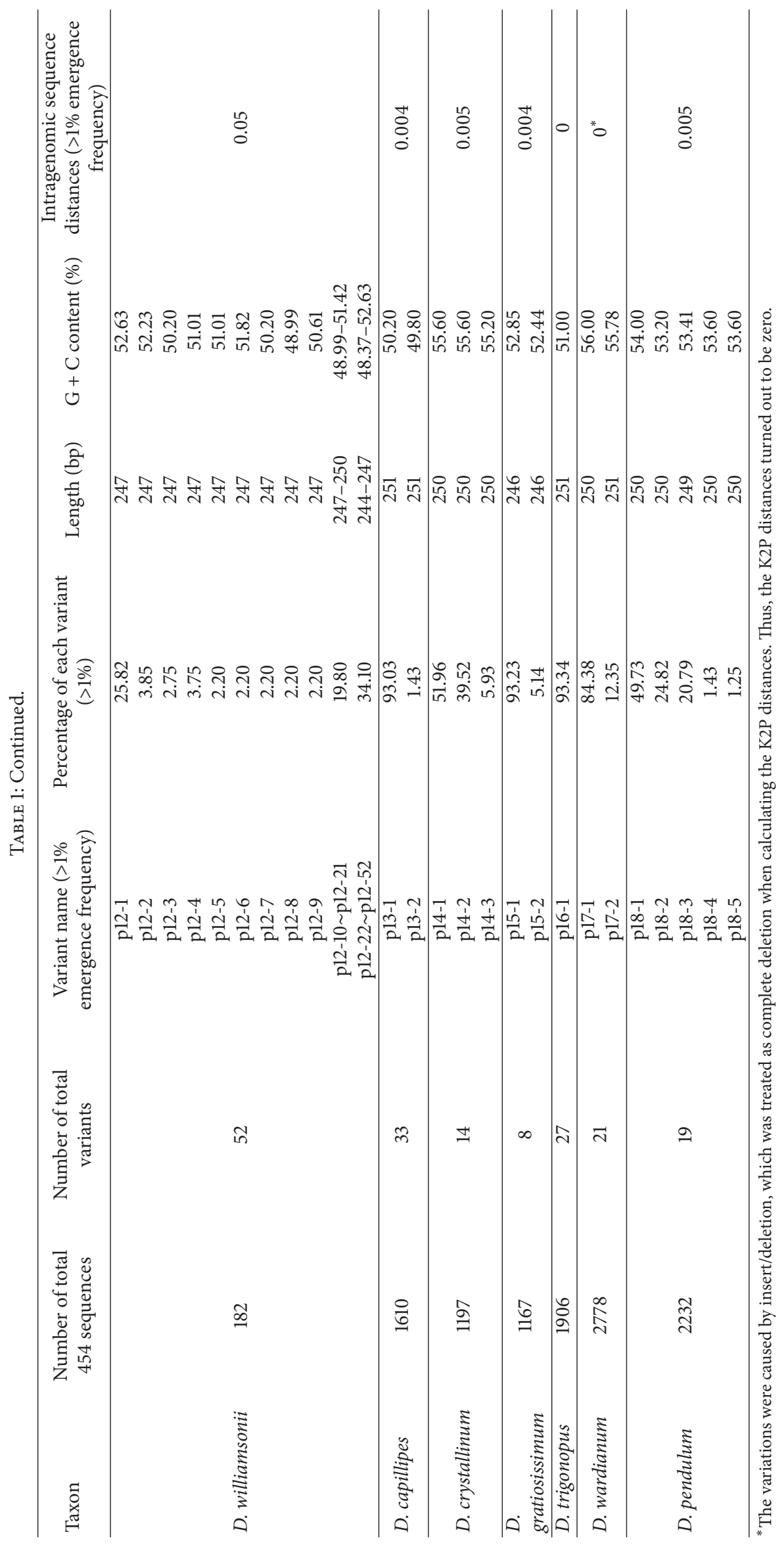


TABLE 2: Comparison between the numbers of intraspecific variable sites with the emergency percentage of the dominant variants in genome of ITS2 sequences of each species.

\begin{tabular}{|c|c|c|c|}
\hline Taxon & $\begin{array}{c}\text { Number of total ITS2 } \\
\text { sequences }\end{array}$ & $\begin{array}{c}\text { Number of total } \\
\text { intraspecific variable sites }\end{array}$ & $\begin{array}{c}\text { The emergency percentage } \\
\text { of the dominant variants in } \\
\text { genome }(\%)\end{array}$ \\
\hline D. crepidatum & 35 & 36 & 99.27 \\
\hline D. aphyllum & 22 & 4 & 95.87 \\
\hline D. devonianum & 11 & 9 & 95.43 \\
\hline D. officinale & 61 & 5 & 93.89 \\
\hline D. denneanum & 10 & 4 & 91.50 \\
\hline D. chrysanthum & 25 & 22 & 76.74 \\
\hline D. primulinum & 21 & 25 & 75.93 \\
\hline D. chrysotoxum & 27 & 18 & 63.82 \\
\hline D. nobile & 59 & 12 & 63.15 \\
\hline D. loddigesii & 13 & 28 & 39.55 \\
\hline
\end{tabular}

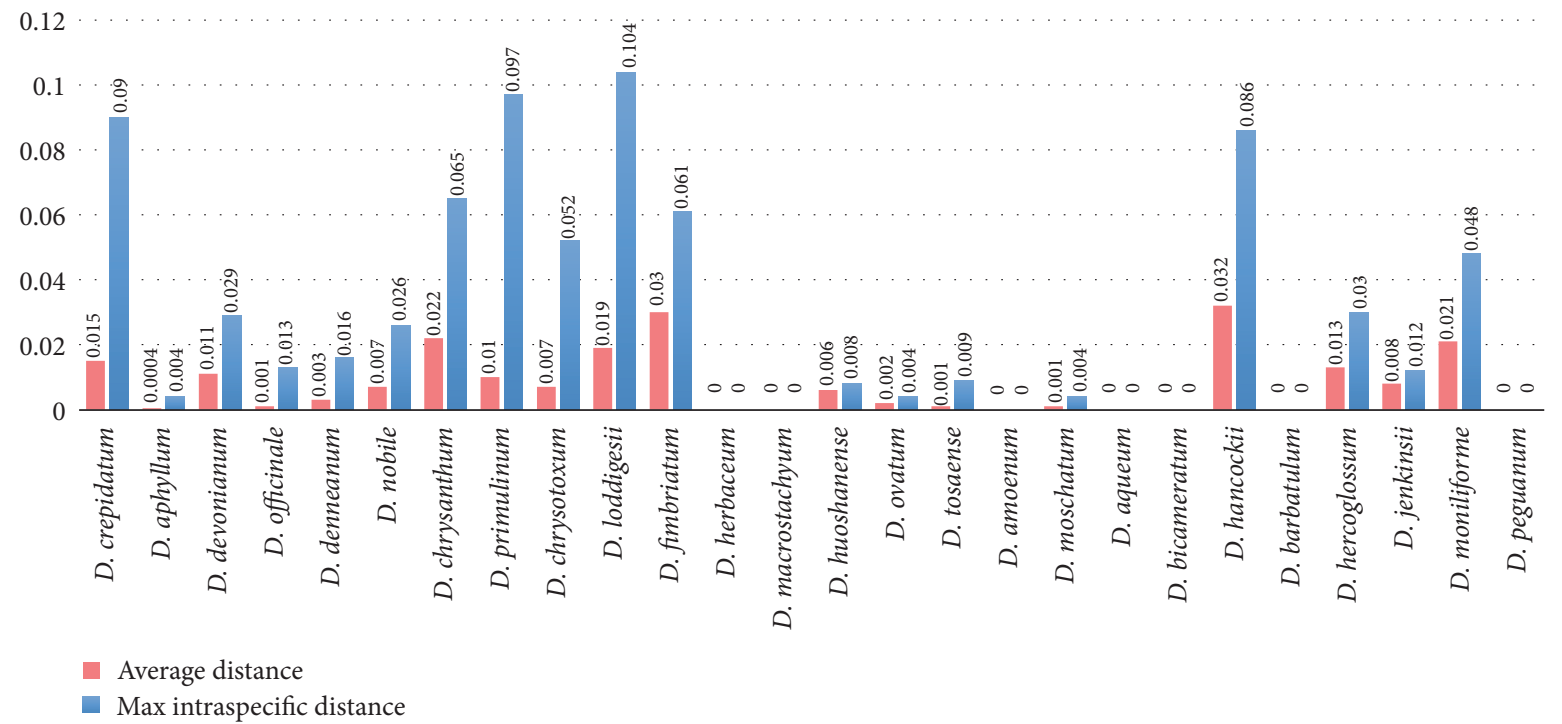

FIGURE 1: Intraspecific genetic distances from 26 Dendrobium species revealed by ITS2 region.

sequence patterns in $D$. officinale were consistent with the sequences obtained via direct PCR sequencing.

\subsection{Analysis of the ITS2 Region at Intra- and Interspecific} Levels. In total, 513 ITS2 sequences from 26 species of Dendrobium were analyzed for intraspecific genetic distances (IS-GDs). The average IS-GD for each species ranged from 0 to 0.032 . The average IS-GD value in seven species $(D$. herbaceum, D. macrostachyum, D. amoenum, D. aqueum, D. bicameratum, D. barbatulum, and D. peguanum) was zero, and the highest average IS-GD value $(0.032)$ was found in D. hancockii (Figure 1). The number of variable sites in ITS2 sequences of each species was also calculated (Table 2). D. officinale possesses a dominant variant representing $93.89 \%$ of the sequences, and out of 61 sequences there were only five intraspecific SNPs. A similar situation was observed in three other species (D. aphyllum, D. devonianum, and $D$. denneanum), all of which had one dominant ITS2 variant making up more than $90 \%$ of sequences. In contrast, there were more variable sites in species where the dominant ITS2 variant made up less than $80 \%$ of the sequences. For example, the dominant variant in $D$. loddigesii accounted for only $39.55 \%$ of sequences, and this species had 28 intraspecific variable sites. In general, as the dominant ITS2 variant became more common in the genome, there were fewer intraspecific variable sites in ITS2 sequences, with the exception of $D$. crepidatum.

The BLAST1 method, which is based on similarity, was used to assess the reliability of ITS2 sequences for Dendrobium species identification. In total, 383 of the 513 ITS2 sequences were correctly identified. The unidentified ITS2 sequences were distributed among six species: D. officinale, $D$. tosaense, D. huoshanense, D. moniliforme, D. nobile, and $D$. hercoglossum. The ITS2 sequences of D. officinale and D. tosaense could not be distinguished using BLAST, and similar issues arose for D. huoshanense, D. moniliforme, D. nobile, and D. hercoglossum. 


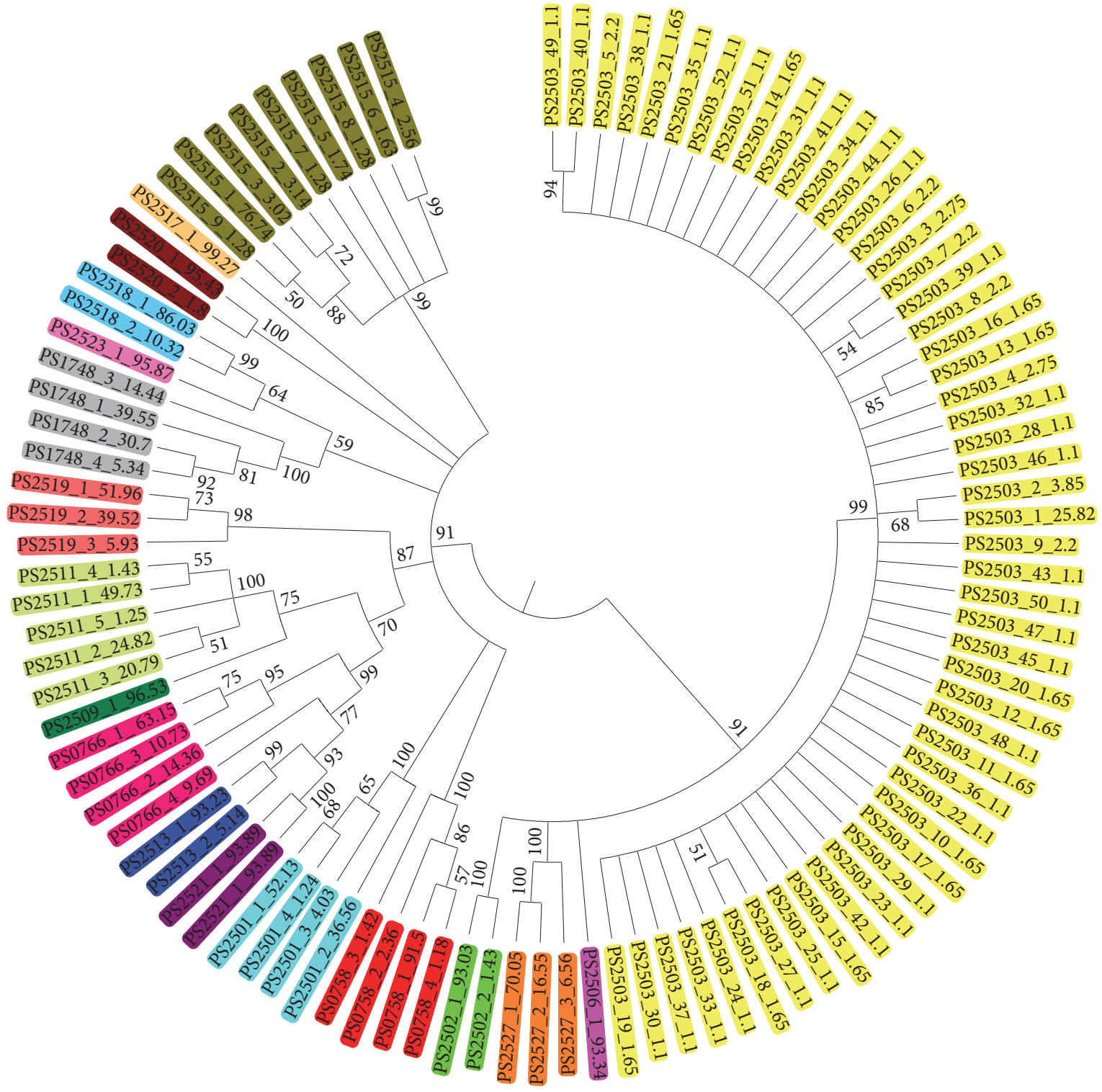

FIgURE 2: The neighbor-joining tree built using the major variants of ITS2 from 18 Dendrobium species. Note. Each species was marked with a unique color. The three parts of the name of each branch represent the voucher number, the variant name of the multiple copy in the genome, and the percentage of its presence in the genome. The taxa with their voucher numbers were listed for the following: D. williamsonii (PS2503), D. trigonopus (PS2506), D. acinaciforme (PS2527), D. capillipes (PS2502), D. loddigesii (PS1748), D. crystallinum (PS2519), D. denneanum (PS0758), D. chrysotoxum (PS2501), D. officinale (PS2521), D. gratiosissimum (PS2513), D. nobile (PS0766), D. wardianum (PS2509), D. pendulum (PS2511), D. aphyllum (PS2523), D. primulinum (PS2518), D. devonianum (PS2520), D. chrysanthum (PS2515), and D. crepidatum (PS2517).

3.3. The Neighbor-Joining Tree Based on ITS2 Sequences. A neighbor-joining tree ( $\mathrm{NJ}$ tree) was built based on the intragenomic data to determine the phylogenetic relationships between the Dendrobium species. Previous studies have shown that minor variants present below $1 \%$ are difficult to detect directly with PCR or clone sequencing. Thus, we first selected the major variants for analysis (Figure 2). The results showed that D. williamsonii (PS2503), D. trigonopus (PS2506), D. acinaciforme (PS2527), and D. capillipes (PS2502) clustered into one clade, with all other species forming a separate clade. Almost all the major variants clustered together, with the exception of D. nobile. One of the major variants of D. nobile (PS0766_4) introgressed into D. officinale and D. gratiosissimum, showing a very close relationship. Another NJ tree using total intragenomic data was also constructed and is shown in Appendix S3.

Total intraspecific data were also used to construct an NJ tree for phylogenetic analysis (see Appendix S4). The results showed that most species were monophyletic except for one clade including six species (D. officinale, D. tosaense, 


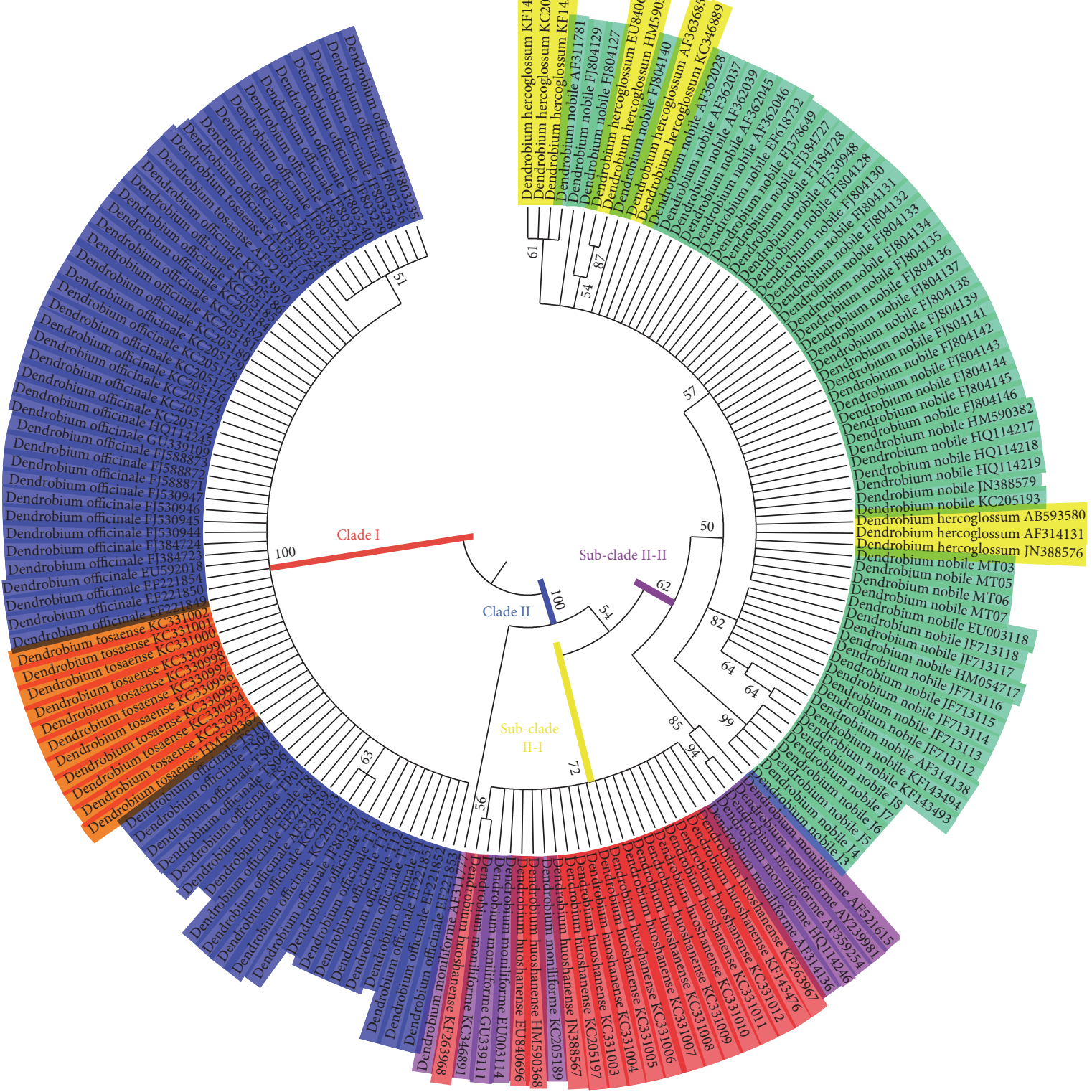

FIGURE 3: The neighbor-joining tree built using ITS2 sequences of the six species. Note. Different colors in Figure 3 represent different species. Blue, D. officinale; red, D. huoshanense; green, D. nobile; purple, D. moniliforme; orange, D. tosaense; yellow, D. hercoglossum.

D. huoshanense, D. moniliforme, D. nobile, and D. hercoglossum). To better clarify the relationship among these species in the main clade, these six species were used to build a separate NJ tree based on their ITS2 sequences (Figure 3). We divided this NJ tree into two major clades (Clades I and II). Clade I consists of two species, $D$. officinale and $D$. tosaense, with a bootstrap support value of $100 \%$. Clade II consists of two subclades, with a bootstrap support value of $54 \%$. Subclades II-I contain two species, D. moniliforme and D. huoshanense, with a bootstrap support value of $72 \%$, and subclades II-II contain three species, D. moniliforme, D. hercoglossum, and D. nobile, with a bootstrap support value of $62 \%$.

\section{Discussion}

Ribosomal DNA (rDNA) is present in multiple copies of tandem repeats per genome [31], and two noncoding spacers (internal transcribed spacer 1 and 2) divide each transcriptional unit into three subunits: 18S, 5.8S and 28S. Each tandem can contain variations, thus leading to intragenomic variation. Many studies have addressed genomic divergence in Dendrobium, but most of these have been focused on intra- and interspecific levels of variation [32-36]. It is thought that biodiversity at the species level is generally overestimated due to intragenomic variation [37]. In this 
study we therefore focused on the intragenomic level, aiming to identify relationships between intragenomic diversity and intraspecific diversity. Sequence-based methods have replaced many traditional approaches such as allozyme or restriction enzyme polymorphisms, which is valid as long as appropriate marker(s) is selected [38]. Traditional approaches (e.g., RAPD, AP-PCR, and AFLP) generally require highquality DNA for amplification, which can lead to problems with reproducibility and accuracy. Sequence-based methods should be more objective and stable, enhancing our ability to assess biodiversity and identify species [39]. In addition, experimental error and subjective factors such as scoring PCR bands on a gel are eliminated or minimized in sequencebased protocols.

The ITS2 locus has already been proposed as a universal DNA barcode, particularly in plants, and it has been shown that plants can be identified at the species and genus level with more than $97 \%$ accuracy $[27,40]$. Although the China plant BOL group suggested ITS as the core barcode for seed plants, ITS2 has several advantages compared with the fulllength ITS region [41]. First, ITS2 is shorter than ITS, which simplifies PCR amplification. Moreover, ITS2 has secondary structure in all eukaryotes $[42,43]$. This molecular morphological characteristic strengthens the power of its discriminatory ability. In addition to species identification applications, ITS2 and its secondary structure have been used as effective tools for phylogenetic analyses in insects, corals, and yeast [44-47]. As these transcribed spacers are highly divergent, they can also be used to estimate low levels of genetic diversity among related species [48]. Liu et al. evaluated the resolution of five regions ( $r b c L$, matK, ITS, ITS2, and $t r n H-p s b A)$, ultimately suggesting an $r b c L$ + ITS2 barcode combination as the most suitable marker for analyzing biodiversity in the Dinghushan National Nature Reserve (DNNR) in China [49].

Among all the Dendrobium species, D. officinale is undoubtedly the most valuable, owing to its low production but high price and clinical efficacy in the clinic. Previous studies using ISSR, RAPD, and SRAP revealed distinct genetic differences and extensive genetic diversity among different populations of $D$. officinale $[34,50,51]$. However, the intraspecific genetic diversity of $D$. officinale (intraspecific genetic distance, average: 0.001; Max: 0.013) as revealed by ITS2 sequences turned out to be relatively low compared with results from other approaches. From the 61 ITS2 sequences obtained from $D$. officinale, only five variable sites were detected after alignment. Across the whole genome, $D$. officinale has a single dominant variant that represents $93.89 \%$ of ITS2 sequences. These results indicate that the ITS2 regions are relatively conserved among different populations of $D$. officinale. Due to the low production and high price of $D$. officinale, there are so many closely related species appearing as adulterants in the herbal market. These adulterants are species that have morphological characteristics similar to each other, making traditional taxonomic identification difficult, particularly after processing into medicinal slices. According to this result above, ITS2 can be an effective molecules tool for identifying commercial $D$. officinale and other Dendrobium species.
In the Chinese Pharmacopoeia (2015 edition), D. officinale is described as an independent species that is the source of the herbal medicine "Tie Pi Shi Hu." However, this species has already been accepted as a synonym of $D$. catenatum, $D$. tosaense, and several others in flora of China and the other research [52]. In our study, ITS2 sequences from these two species were grouped into a single clade with $100 \%$ bootstrap support. The NJ tree described here demonstrates that, at the very least, $D$. officinale and $D$. tosaense are extremely closely related at the genetic level, consistent with other results from China. Therefore, we agree that D. officinale and $D$. tosaense should be accepted as synonyms of D. catenatum. In a previous study, a phylogenetic tree including twelve samples of Dendrobium species was constructed [50]. The three species $D$. moniliforme, $D$. hercoglossum, and $D$. nobile were grouped in the same clade, similar to classifications based on inflorescence color and the results from this study.

\section{Conclusion}

In this study, we analyzed intragenome and intraspecies divergence to find that, in most cases, greater intragenomic divergence is correlated with larger intraspecific variation. The results of this study strongly confirm that the direct PCR sequencing data were credible because all the dominant sequences in high-throughput sequencing in each species were detected by direct PCR. Thus, the multiple copies in ITS2 did not affect the species identification in Dendrobium. Therefore, we demonstrate that ITS2 is an effective tool for Dendrobium species identification.

\section{Abbreviations}

$\begin{array}{ll}\text { ITS2: } & \text { Internal transcribed spacer } 2 \\ \text { rDNA: } & \text { Ribosomal DNA } \\ \text { IS-GD: } & \text { Intraspecific genetic distances } \\ \text { ITS: } & \text { Internal transcribed spacer } \\ \text { IG-GD: } & \text { Intragenomic genetic distance } \\ \text { SNP: } & \text { Single nucleotide polymorphisms } \\ \text { HMM: } & \text { Hidden Markov model } \\ \text { NJ tree: } & \text { Neighbor-joining tree } \\ \text { PCR: } & \text { Polymerase chain reaction } \\ \text { bp: } & \text { Base pair } \\ \text { INDELS: } & \text { Insertions and deletions } \\ \text { RAPD: } & \text { Random amplified polymorphic DNA } \\ \text { AP-PCR: } & \text { Arbitrarily primed PCR } \\ \text { AFLP: } & \text { Amplified fragment length polymorphism } \\ \text { matK: } & \text { Maturase K } \\ \text { rbcL: } & \text { Ribulose-bisphosphate carboxylase gene } \\ \text { trnH-psbA: } & \text { Intergenic spacer } \\ \text { ISSR: } & \text { Inter-simple sequence repeat } \\ \text { SRAP: } & \text { Sequence-related amplified polymorphism. }\end{array}$

\section{Additional Points}

Supporting Information. DNA sequences can be obtained through GenBank accessions; final DNA sequence assembly can be found in online supporting information. 


\section{Conflicts of Interest}

The authors declare that there are no conflicts of interest regarding the publication of this article.

\section{Authors' Contributions}

Jianping Han designed the research, Xiaochen Chen conducted the experimental work, Lili Wang analyzed data, and Xiaoyue Wang wrote the manuscript.

\section{Acknowledgments}

This work was supported by grants from the National Natural Science Foundation of China (no. 81473303) and the Major Scientific and Technological Special Project for "Significant New Drugs Creation” (no. 2014ZX09304307001). The authors thank Professor Yulin Lin for identifying material.

\section{References}

[1] X. Y. Ding, W. M. Zhang, Z. T. Wang, and L. S. Xu, "Summarization of the studies of taxonomy and pharmacognosy of ethnological materials of Dendrobium," The Chinese Academic Medical Magazine of Organisms, no. 1, pp. 1-14, 2003.

[2] S. Y. Yang, S. G. Wang, H. L. Li, and Z. H. Di, "Research Status of Identification on CAULIS DENDROBII," Journal of Anhui Agricultural Sciences, vol. 41, pp. 1495-1497, 2013.

[3] S. O. Rogers and A. J. Bendich, "Ribosomal RNA genes in plants: variability in copy number and in the intergenic spacer," Plant Molecular Biology, vol. 9, no. 5, pp. 509-520, 1987.

[4] D. M. Hillis and M. T. Dixon, "Ribosomal DNA: molecular evolution and phylogenetic inference," The Quarterly Review of Biology, vol. 66, no. 4, pp. 411-453, 1991.

[5] C. D. Prokopowich, T. R. Gregory, and T. J. Crease, "The correlation between rDNA copy number and genome size in eukaryotes," Genome, vol. 46, no. 1, pp. 48-50, 2003.

[6] F. J. Stewart and C. M. Cavanaugh, "Intragenomic variation and evolution of the internal transcribed spacer of the rRNA operon in bacteria," Journal of Molecular Evolution, vol. 65, no. 1, pp. 4467, 2007.

[7] C. Li and R. C. Wilkerson, "Intragenomic rDNA ITS2 variation in the neotropical Anopheles (Nyssorhynchus) albitarsis complex (Diptera: Culicidae)," Journal of Heredity, vol. 98, no. 1, pp. 51-59, 2007.

[8] D. J. Harris and K. A. Crandall, "Intragenomic variation within ITS1 and ITS2 of freshwater crayfishes (Decapoda: Cambaridae): Implications for phylogenetic and microsatellite studies," Molecular Biology and Evolution, vol. 17, no. 2, pp. 284-291, 2000.

[9] M. He, L. Huang, J. Shi, and Y. Jiang, "Variability of ribosomal DNA ITS-2 and its utility in detecting genetic relatedness of pearl oyster," Marine Biotechnology, vol. 7, no. 1, pp. 40-45, 2005.

[10] R. Freire, A. Arias, J. Méndez, and A. Insua, "Sequence variation of the internal transcribed spacer (ITS) region of ribosomal DNA in Cerastoderma species (Bivalvia: Cardiidae)," Journal of Molluscan Studies, vol. 76, no. 1, pp. 77-86, 2010.

[11] Z.-L. Liu, D. Zhang, X.-Q. Wang, X.-F. Ma, and X.-R. Wang, "Intragenomic and interspecific 5S rDNA sequence variation in five Asian pines," American Journal of Botany, vol. 90, no. 1, pp. 17-24, 2003.
[12] S. Feng, Y. Jiang, S. Wang et al., "Molecular identification of dendrobium species (Orchidaceae) based on the dna barcode its2 region and its application for phylogenetic study," International Journal of Molecular Sciences, vol. 16, no. 9, pp. 21975-21988, 2015.

[13] S. Xu, D. Li, J. Li et al., "Evaluation of the DNA barcodes in dendrobium (Orchidaceae) from mainland Asia," PLoS ONE, vol. 10, no. 1, Article ID e0115168, 2015.

[14] X.-Y. Ding, Z.-T. Wang, H. Xu, L.-S. Xu, and K.-Y. Zhou, "Database establishment of the whole rDNA its region of Dendrobium species of "Fengdou" and authentication by analysis of their sequences," Yaoxue Xuebao, vol. 37, no. 7, pp. 567-573, 2002.

[15] L. Zhang, D. S. Qiu, S. K. Cai, R. Y. Deng, H. M. Luo, and X. J. Liu, "The Genuine and Adulterants Identification of Dendrobium officinaleby rDNA ITS Sequences Analysis," Journal of Anhui Agricultural Sciences, vol. 4, pp. 2872-2874, 2013.

[16] D. Li, J. Z. Li, P. Mao, F. Y. X, Z. Chun, and X. R. Ma, "Phylogenetic Analysis and Identification of Dendrobium Species Based on Ribosomal DNA Internal Transcribed Spacer (ITS) sequence," Acta Horticulturae Sinica, vol. 39, pp. 1539-1550, 2012.

[17] I. Álvarez and J. F. Wendel, "Ribosomal ITS sequences and plant phylogenetic inference," Molecular Phylogenetics and Evolution, vol. 29, no. 3, pp. 417-434, 2003.

[18] G. N. Feliner and J. A. Rosselló, "Better the devil you know? Guidelines for insightful utilization of nrDNA ITS in specieslevel evolutionary studies in plants," Molecular Phylogenetics and Evolution, vol. 44, no. 2, pp. 911-919, 2007.

[19] L.-C. Shi, J. Zhang, J.-P. Han et al., "Testing the potential of proposed DNA barcodes for species identification of Zingiberaceae," Journal of Systematics and Evolution, vol. 49, no. 3, pp. 261-266, 2011.

[20] X. Chen, B. Liao, J. Song, X. Pang, J. Han, and S. Chen, "A fast SNP identification and analysis of intraspecific variation in the medicinal Panax species based on DNA barcoding," Gene, vol. 530, no. 1, pp. 39-43, 2013.

[21] X. Z. Zhu and T. Gao, "Molecular identification of 17 invasive plant species based on ITS2 sequences," Pratacultural Science, vol. 31, pp. 1900-1907, 2014.

[22] J. M. Guillamön, J. Sabaté, E. Barrio, J. Cano, and A. Querol, "Rapid identification of wine yeast species based on RFLP analysis of the ribosomal internal transcribed spacer (ITS) region," Archives of Microbiology, vol. 169, no. 5, pp. 387-392, 1998.

[23] Y. C. Chen, J. D. Eisner, M. M. Kattar et al., "Identification of medically important yeasts using PCR-based detection of DNA sequence polymorphisms in the internal transcribed spacer 2 region of the rRNA genes," Journal of Clinical Microbiology, vol. 38 , no. 6, pp. 2302-2310, 2000.

[24] L. Xiang, J. Song, T. Xin et al., "DNA barcoding the commercial Chinese caterpillar fungus," FEMS Microbiology Letters, vol. 347, no. 2, pp. 156-162, 2013.

[25] R. Stouthamer, J. Hu, F. J. P. M. Van Kan, G. R. Platner, and J. D. Pinto, "The utility of internally transcribed spacer 2 DNA sequences of the nuclear ribosomal gene for distinguishing sibling species of Trichogramma," BioControl, vol. 43, no. 4, pp. 421-440, 1999.

[26] W. J. Kress, K. J. Wurdack, E. A. Zimmer, L. A. Weigt, and D. H. Janzen, "Use of DNA barcodes to identify flowering plants," Proceedings of the National Acadamy of Sciences of the United States of America, vol. 102, no. 23, pp. 8369-8374, 2005. 
[27] S. L. Chen, H. Yao, J. P. Han et al., "Validation of the ITS2 region as a novel DNA barcode for identifying medicinal plant species," PLoS ONE, vol. 5, no. 1, Article ID e8613, 2010.

[28] J. Song, L. Shi, D. Li et al., "Extensive pyrosequencing reveals frequent intra-genomic variations of internal transcribed spacer regions of nuclear ribosomal DNA," PLoS ONE, vol. 7, no. 8, Article ID e43971, 2012.

[29] A. Keller, T. Schleicher, J. Schultz, T. Müller, T. Dandekar, and M. Wolf, "5.8S-28S rRNA interaction and HMM-based ITS2 annotation," Gene, vol. 430, no. 1-2, pp. 50-57, 2009.

[30] K. Tamura, D. Peterson, N. Peterson, G. Stecher, M. Nei, and S. Kumar, "MEGA5: molecular evolutionary genetics analysis using maximum likelihood, evolutionary distance, and maximum parsimony methods," Molecular Biology and Evolution, vol. 28, no. 10, pp. 2731-2739, 2011.

[31] K. Beckingham, "Insect rDNA," The Cell Nucleus, vol. 10, pp. 205-269, 1982.

[32] H. H. Fan, T. C. Li, J. Qiu, Z. P. Li, Y. Lin, and Y. P. Cai, "Studies on genetic diversity of medicinal Dendrobium by SRAP," Zhongguo Zhong Yao Za Zhi, vol. 33, pp. 6-10, 2008.

[33] M. Sharif and Abdul Jawad, "Interacting generalized dark energy and reconstruction of scalar field models," Modern Physics Letters A, vol. 28, no. 38, Article ID 1350180, 15 pages, 2013.

[34] Y. H. Bao, C. M. Pan, Y. Bai, and Y. N. Yan, "Genetic diversity of germplasm resources of Tiepishihu (Dendrobium officinale) analyzed with SRAP," Journal of Beijing University of Chinese Medicine, vol. 37, pp. 349-353, 2014.

[35] Y. T. Liu, R. K. Chen, S. J. Lin et al., "Analysis of sequence diversity through internal transcribed spacers and simple sequence repeats to identify dendrobium species," Genetics and Molecular Research, vol. 13, no. 2, pp. 2709-2717, 2014.

[36] W. L. Zhang, "Page analysis of genetic diversity of Dendrobium germplasm resources," Northern Horticulture, pp. 104-106, 2014.

[37] D. J. Thornhill, T. C. Lajeunesse, and S. R. Santos, "Measuring rDNA diversity in eukaryotic microbial systems: How intragenomic variation, pseudogenes, and PCR artifacts confound biodiversity estimates," Molecular Ecology, vol. 16, no. 24, pp. 5326-5340, 2007.

[38] M. Hajibabaei, G. A. C. Singer, P. D. N. Hebert, and D. A. Hickey, "DNA barcoding: how it complements taxonomy, molecular phylogenetics and population genetics," Trends in Genetics, vol. 23, no. 4, pp. 167-172, 2007.

[39] R. M. Caesar, M. Sörensson, and A. I. Cognato, "Integrating DNA data and traditional taxonomy to streamline biodiversity assessment: An example from edaphic beetles in the Klamath ecoregion, California, USA," Diversity and Distributions, vol. 12, no. 5, pp. 483-489, 2006.

[40] H. Yao, J. Song, C. Liu et al., "Use of ITS2 region as the universal DNA barcode for plants and animals," PLoS ONE, vol. 5, no. 10, Article ID e13102, 2010.

[41] J. Han, Y. Zhu, X. Chen et al., "The short ITS2 sequence serves as an efficient taxonomic sequence tag in comparison with the fulllength ITS," BioMed Research International, vol. 2013, Article ID 741476, 7 pages, 2013.

[42] J. C. Mai and A. W. Coleman, “The internal transcribed spacer 2 exhibits a common secondary structure in green algae and flowering plants," Journal of Molecular Evolution, vol. 44, no. 3, pp. 258-271, 1997.

[43] J. Schultz, S. Maisel, D. Gerlach, T. Müller, and M. Wolf, "A common core of secondary structure of the internal transcribed spacer 2 (ITS2) throughout the Eukaryota," $R N A$, vol. 11, no. 4, pp. 361-364, 2005.

[44] T. J. Lott, B. M. Burns, R. Zancope-Oliveira, C. M. Elie, and E. Reiss, "Sequence analysis of the internal transcribed spacer 2 (ITS2) from yeast species within the genus Candida," Current Microbiology, vol. 36, no. 2, pp. 63-69, 1998.

[45] H.-E. Lein, C.-F. Dai, and C. C. Wallace, "Secondary structure and phylogenetic utility of the ribosomal internal transcribed spacer 2 (ITS2) in scleractinian corals," Zoological Studies, vol. 43, pp. 759-771, 2004.

[46] I. Young and A. W. Coleman, "The advantages of the ITS2 region of the nuclear rDNA cistron for analysis of phylogenetic relationships of insects: A Drosophila example," Molecular Phylogenetics and Evolution, vol. 30, no. 1, pp. 236-242, 2004.

[47] J. Schultz, T. Müller, M. Achtziger, P. N. Seibel, T. Dandekar, and M. Wolf, "The internal transcribed spacer 2 database - A web server for (not only) low level phylogenetic analyses," Nucleic Acids Research, vol. 34, pp. W704-W707, 2006.

[48] G. N. Fritz, J. Conn, A. Cockburn, and J. Seawright, "Sequence analysis of the ribosomal DNA internal transcribed spacer 2 from populations of Anopheles nuneztovari (diptera: culicidae)," Molecular Biology and Evolution, vol. 11, no. 3, pp. 406416, 1994.

[49] J. Liu, H.-F. Yan, S. G. Newmaster, N. Pei, S. Ragupathy, and X.J. Ge, "The use of DNA barcoding as a tool for the conservation biogeography of subtropical forests in China," Diversity and Distributions, vol. 21, no. 2, pp. 188-199, 2015.

[50] G. Ding, X.-Y. Ding, J. Shen et al., "Genetic diversity and molecular authentication of wild populations of Dendrobium officinale by RAPD," Yaoxue Xuebao, vol. 40, no. 11, pp. 10281032, 2005.

[51] J. S. Lu, Z. Y. Bu, W. L. Lv, J. M. Su, C. Y. Huang, and C. N. Li, "ISSR analysis on genetic diversity of germplasms resources in Dendrobium SW. from different habitats," Chinese Traditional and Herbal Drugs44, vol. 44, pp. 96-100, 2013.

[52] T. Takamiya, P. Wongsawad, N. Tajima et al., "Identification of Dendrobium species used for herbal medicines based on ribosomal DNA Internal transcribed spacer sequence," Biological \& Pharmaceutical Bulletin, vol. 34, no. 5, pp. 779-782, 2011. 

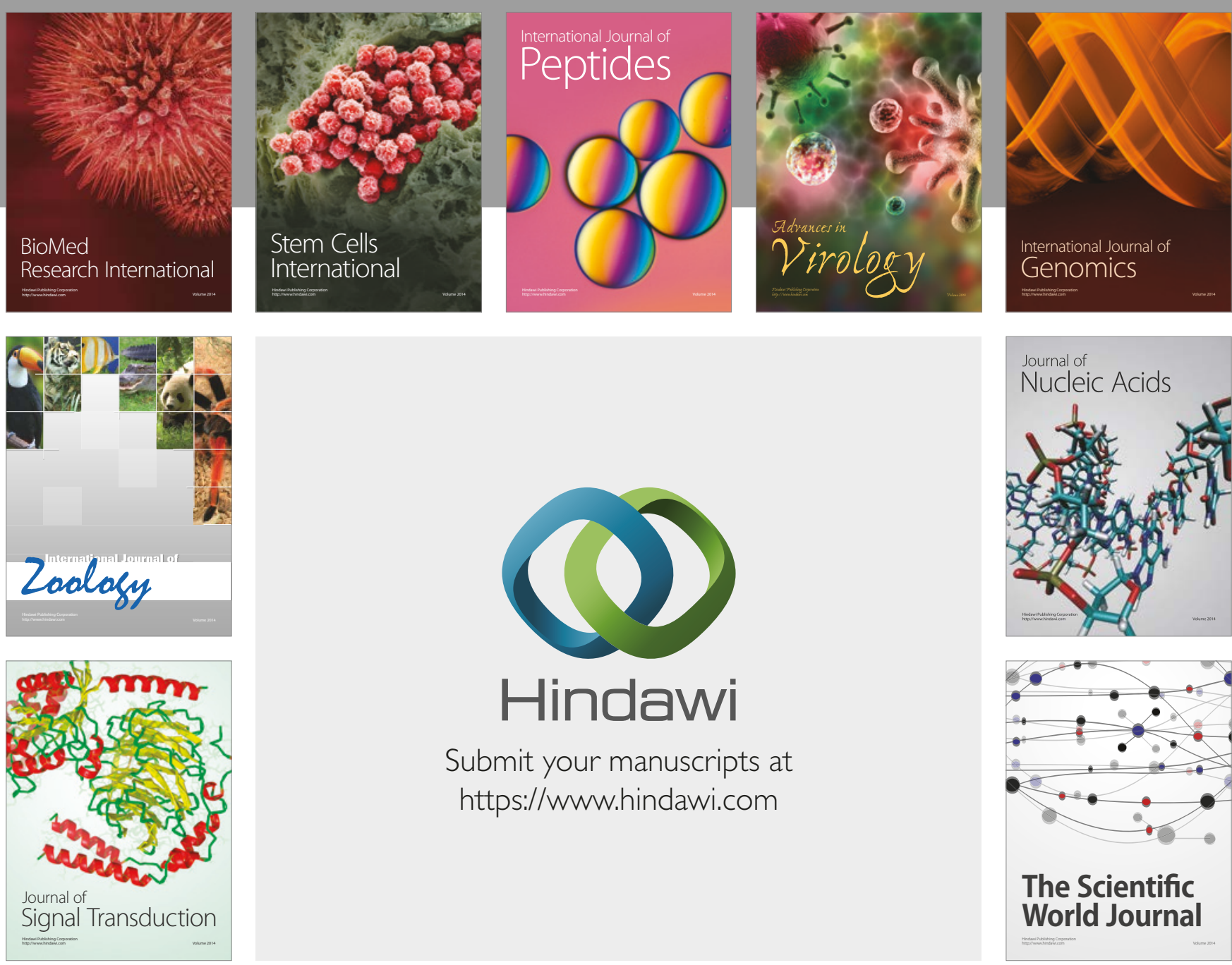

Submit your manuscripts at

https://www.hindawi.com
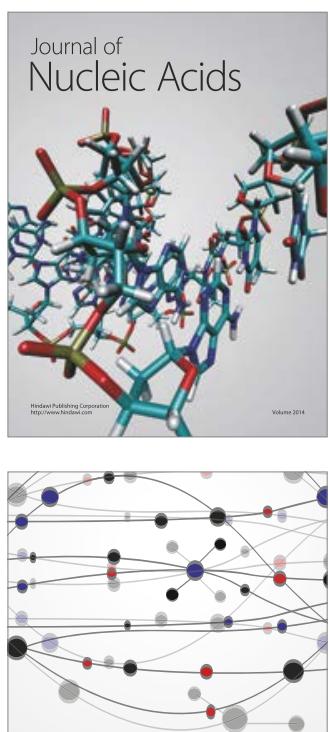

The Scientific World Journal

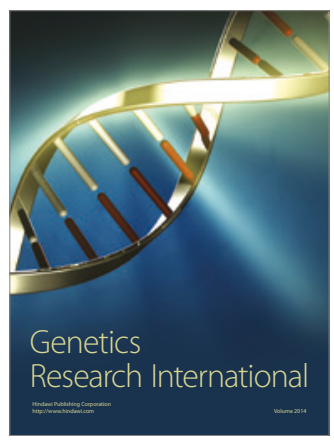

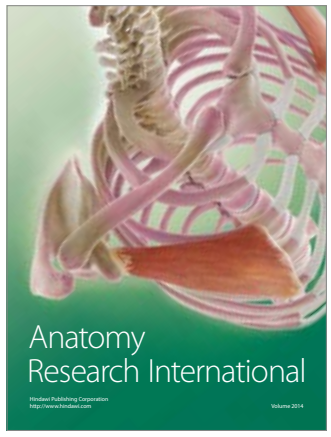

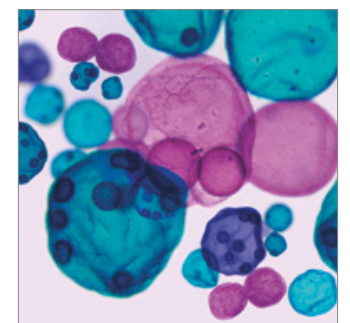

International Journal of Microbiology
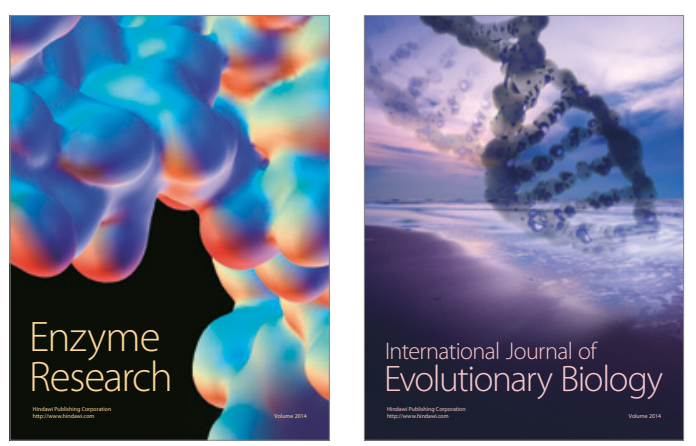
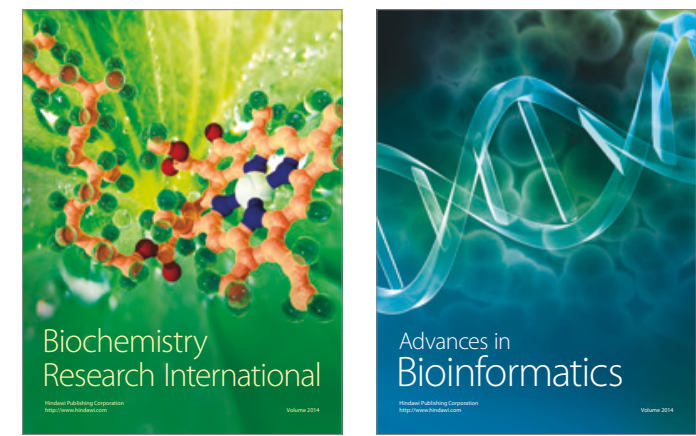

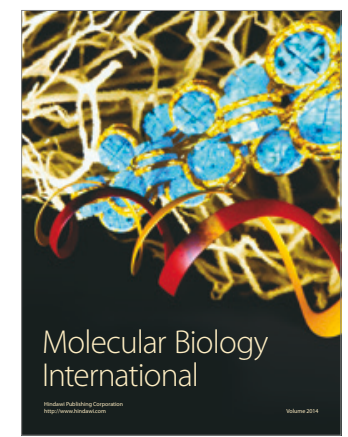

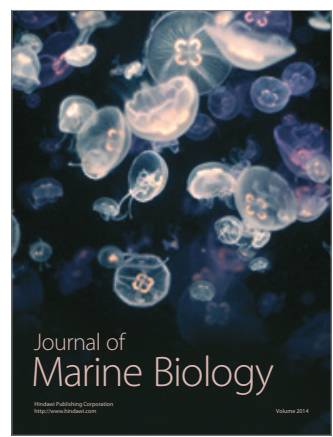

